\title{
First observation of a stable highly-dissipative divertor plasma regime on the Wendelstein 7-X stellarator
}

\author{
D. Zhang, ${ }^{1}$ R. König, ${ }^{1}$ Y. Feng, ${ }^{1}$ R. Burhenn, ${ }^{1}$ S. Brezinsek, ${ }^{2}$ M. Jakubowski, ${ }^{1}$ B. Buttenschön, ${ }^{1}$ \\ H. Niemann, ${ }^{1}$ A. Pavone, ${ }^{1}$ M. Krychowiak, ${ }^{1}$ S. Kwak, ${ }^{1}$ J. Svensson, ${ }^{1}$ Y. Gao, ${ }^{2}$ T.S. Pedersen, ${ }^{1}$ \\ A. Alonso, ${ }^{3}$ J. Baldzuhn, ${ }^{1}$ C.D. Beidler,${ }^{1}$ C. Biedermann, ${ }^{1}$ S. Bozhenkov, ${ }^{1}$ K.J. Brunner, ${ }^{1}$ H. \\ Damm, ${ }^{1}$ M. Hirsch, ${ }^{1}$ L. Giannone, ${ }^{4}$ P. Drewelow, ${ }^{1}$ F. Effenberg, ${ }^{5}$ G. Fuchert, ${ }^{1}$ K.C. Hammond, ${ }^{1}$ U. \\ Höfel, ${ }^{1}$ C. Killer, ${ }^{1}$ J. Knauer ${ }^{1}$ H.P. Laqua, ${ }^{1}$ R. Laube, ${ }^{1}$ N. Pablant,${ }^{6}$ E. Pasch, ${ }^{1}$ F. Penzel, ${ }^{4}$ K. \\ Rahbarnia, ${ }^{1}$ F. Reimold,${ }^{1}$ H. Thomsen,${ }^{1}$ V. Winters, ${ }^{5}$ F. Wagner, ${ }^{1}$ T. Klinger, ${ }^{1}$ and the W7-X team ${ }^{1}$ \\ ${ }^{1}$ Max-Planck-Institut für Plasmaphysik, 17491 Greifswald, Germany \\ ${ }^{2}$ Forschungszentrum Jülich GmbH, IEK-4 52425 Juelich, Germany \\ ${ }^{3}$ Laboratorio Nacional de Fusión - CIEMAT, 28040 Madrid, Spain \\ ${ }^{4}$ Max-Planck-Institut für Plasmaphysik, 85748 Garching, Germany \\ ${ }^{5}$ University of Wisconsin - Madison, Wisconsin 53706, USA \\ ${ }^{6}$ Princeton Plasma Physics Laboratory, Princeton, NJ 08540, USA
}

(Dated: May 26, 2019)

\begin{abstract}
For the first time, the optimized stellarator Wendelstein 7-X has operated with an island divertor. An operation regime in hydrogen was found in which the total plasma radiation approached the absorbed heating power without noticeable loss of stored energy. The divertor thermography recorded simultaneously a strong reduction of the heat load on all divertor targets, indicating almost complete power detachment. This operation regime was stably sustained over several energy confinement times until the pre-programmed end of the discharge. The plasma radiation is mainly due to oxygen and is located at the plasma edge. This plasma scenario is reproducible and robust at various heating powers, plasma densities and gas fueling locations. These experimental results show that the island divertor concept actually works and displays good power dissipation potential, producing a promising exhaust concept for the stellarator reactor line.
\end{abstract}

Reduction of the heat flux density on plasma facing components to a technically feasible level is one of the most challenging issues for magnetic confinement fusion devices on the path to a reactor. A promising approach is the creation of a controlled impurity radiation zone in the vicinity of the plasma-surface-interaction (PSI) area by means of a divertor[1-8]. The basic idea of a divertor configuration[9-11] is to separate the PSI region from the plasma confinement region by establishing an appropriate separatrix-bounded magnetic configuration. In the advanced stellarator Wendelstein 7-AS (W7AS)[12] and the optimized stellarator Wendelstein 7-X (W7-X) [13, 14], this separation is realized by an intrinsic, low-order magnetic island chain which forms the basis for the so-called island divertor configuration. The island divertor concept has been successfully tested on W7-AS. In particular heat flux reduction onto divertor targets with 'partial' plasma detachment has been observed[15-18]. W7-X is an optimized quasi-isodynamic stellarator[19] with shaped non-planar superconducting coils. It is designed for long-pulse steady-state plasma operation with the goal of bringing the optimized stellarator to reactor maturity. Development of a reactor-relevant island divertor concept is one of the main scientific objectives of W7-X [20]. The essential question is the following: How much power can be removed via line radiation of low- $\mathrm{Z}$ impurities without serious impurity contamination and degradation of energy confinement at the core plasma? Plasma detachment from the divertor targets can play a decisive role here. In this paper, we present first observations of a stable, highly-radiative divertor regime, in which the radiation power loss approaches the absorbed heating power, leading to almost complete power detachment while the stored plasma energy is preserved. We note the difference between the stable detachment on W7-AS and W7-X: Most significant is that the heat flux reduction on the targets in W7-AS is inhomogeneous and a certain target range still receives a notable heat flux even at the maximum radiation level and is referred to as "partial" detachment[15-18]. By contrast, the detachment achieved in W7-X is more complete in the sense that the heat flux reduces uniformly on all the targets. W7-X (major radius of $5.5 \mathrm{~m}$, effective minor radius $0.5 \mathrm{~m}$, and plasma volume $30 \mathrm{~m}^{3}$ ) is currently the largest advanced stellarator worldwide. After initial operation with a limiter configuration[21-23], the device was subsequently equipped with ten uncooled graphite divertor units (figure 1(a)), presently being replaced by corresponding water-cooled divertor modules. The first wall (made of stainless steel) is partially covered with graphite tiles in regions exposed to high thermal loads $\left(>0.3 \mathrm{MW} / \mathrm{m}^{2}\right)$. Wall conditioning was done by means of glow- and ECRH-discharges in helium (boronization became possible only after this initial divertor campaign). The plasma is generated by electron cyclotron resonance heating (ECRH). Carbon and oxygen have been identified as the main impurity species. The experiments described here were carried out in a magnetic configuration 
with a $5 / 5$ island chain at the plasma edge and $2.52 \mathrm{~T}$ magnetic field on axis. The island chain has an effective radial dimension of about $6.5 \mathrm{~cm}[24]$.
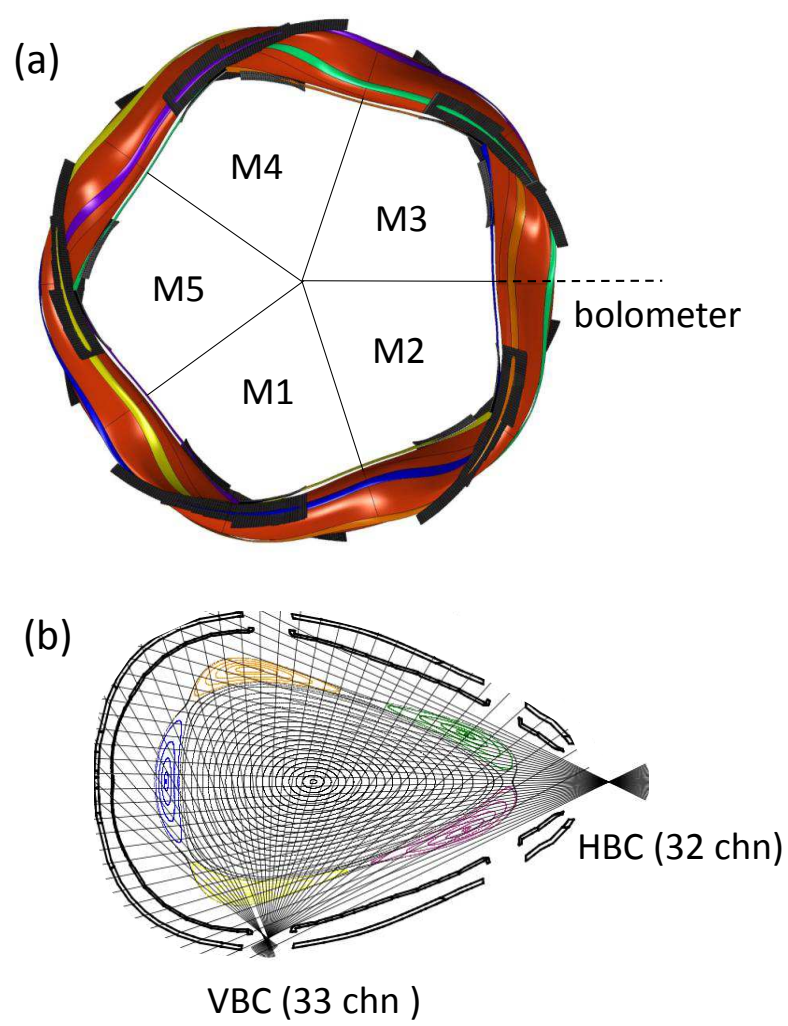

FIG. 1. (a) The stellarator W7-X equipped with ten graphite divertor units. The islands in the scrape-off layer (SOL) intersected by the divertor elements. (b) The lines of sight of the horizontal and vertical bolometer cameras (HBC and VBC) installed at the triangular cross-section for monitoring the plasma radiation from both confinement region and SOL. The Poincaré plot of the magnetic flux surfaces at this cross-section is also shown).

Plasma radiation is measured by two bolometer cameras installed at a triangular cross-section of W7-X with a spatial resolution of $3-4 \mathrm{~cm}$ (figure $1(\mathrm{~b}))[25]$. No divertor modules are installed at this toroidal position. To obtain the total radiated power loss, $P_{\text {rad, }}$ the radiation from the observation volume is extrapolated to the whole plasma volume. Possible toroidal variations of the radiation strength are not considered, as supported by EMC3-EIRENE[26] calculations at different toroidal positions. Although a tomographic reconstruction is possible, the line-integrated signals are used for $P_{\text {rad }}$ calculations in order to avoid uncertainties from the deconvolution procedure. Other plasma parameters and diagnostics involved [27-29] are: an interferometer for the line-averaged plasma density, a diamagnetic loop for the plasma stored energy, an electron cyclotron emission (ECE) radiometer for electron tempera- ture, a Thomson scattering (TS) system for electron density and temperature profile, infrared (IR)-cameras for divertor thermography measuring the divertor heat load, and spectroscopic diagnostics for impurity line emissions and bremsstrahlung radiation. The Minerva Bayesian modelling framework[30] has been used for fitting the TS measurements and deriving the effective ion charge $Z_{\text {eff }}$ based on the bremsstrahlung radiation.

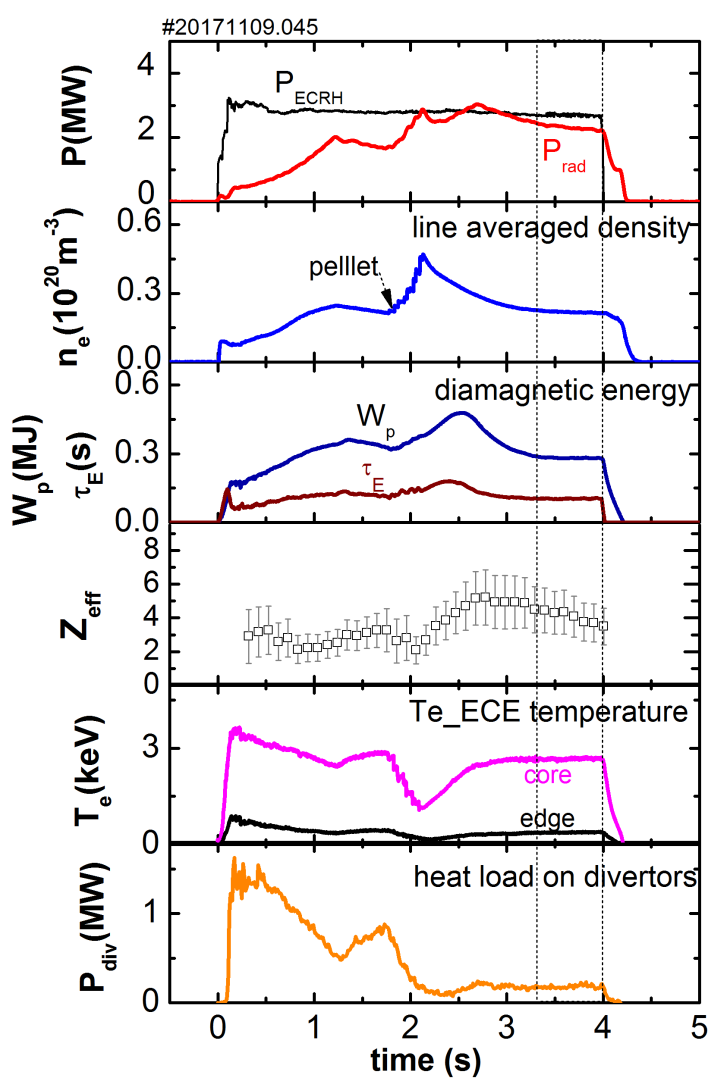

FIG. 2. Time traces of plasma parameters of discharge \#20171109.045. From top to bottom: ECR-heating power $P_{\mathrm{ECRH}}$ and total radiation power $P_{\mathrm{rad}}$, line-averaged density, stored energy $W_{\mathrm{P}}$ and energy confinement time $\tau_{\mathrm{E}}$, the effective ion charge $Z_{\text {eff }}$, two representative ECE channels for respective core and edge electron temperature $T_{\mathrm{e}}$, total heat load on all targets. Pellets are injected in the time interval from 1.7 to $2.1 \mathrm{~s}$.

Fig. 2 shows diagnostic time traces for the hydrogen discharge \#20171109.045, in which at $t>2 \mathrm{~s}$ a highlyradiative regime is established with a quasi-stationary phase between $t=3.3$ and $4 \mathrm{~s}$. At constant ECRH power of $\sim 3 \mathrm{MW}$, a plasma density of $2.5 \times 10^{19} \mathrm{~m}^{-3}$ is first obtained via gas-puffing ( $t=0$ to $1.2 \mathrm{~s}$ ) and afterwards further increased by repetitively injecting hydrogen pellets (from $1.7 \mathrm{~s}$ to $2.1 \mathrm{~s}$ ). Shortly after pellet injection, the stored energy $W_{\mathrm{p}}$ reaches a maximum (cf. also on the stellarator $\operatorname{LHD}[31,32])$, followed by a decay on a time scale larger than the energy confinement time $\tau_{\mathrm{E}}$ of 0.15 $\mathrm{s}$, but corresponding to the particle confinement time $\tau_{\mathrm{P}}$ 
of around $0.5 \mathrm{~s}$. After the initial transient phase for $t>$ $3 \mathrm{~s}$, the line-averaged plasma density as well as $W_{\mathrm{P}}$ and $\tau_{\mathrm{E}}$ approach the levels prior to pellet injection. The total radiation $P_{\text {rad }}$, however, does not return to its pre-pellet value but stays at a higher level close to the total heating power $P_{\mathrm{ECRH}}$, with a fraction $f_{\mathrm{rad}}=P_{\mathrm{rad}} / P_{\mathrm{ECRH}}=90 \%$ (estimated error $\pm 10 \%$ ). Meanwhile, the total heat load on all divertor targets obtained from IR-camera measurements (integrated over the entire divertor surface) drops strongly to $P_{\text {div }} \sim 0.2 \mathrm{MW}$, corresponding to a heat flux of $0.15 \mathrm{MW} / \mathrm{m}^{2}$. For comparison before pellet injection, its maximum value was $\sim 3 \mathrm{MW} / \mathrm{m}^{2}$. This drop of the heat load (by a factor of more than 10) to a level slightly above the diagnostic resolution indicates almost complete power detachment. The full recovery of the core plasma is seen in the time evolution of $T_{\mathrm{e}}$ and the evolution of electron temperature and density profiles measured with Thomson scattering (figure 3 ). From $t=3.3 \mathrm{~s}$ on, the plasma reaches a quasi-stationary, highly radiative divertor plasma regime, during which the plasma stored energy $W_{\mathrm{P}}$ remains constant at $W_{\mathrm{P}} \sim 280 \mathrm{~kJ}$. The global energy confinement time $\tau_{\mathrm{E}} \approx W_{\mathrm{P}} / P_{\mathrm{ECRH}}$ is slightly above $0.1 \mathrm{~s}$, almost the same as that before pellet injection. This regime is self-sustained over several energy confinement times terminated only by the preprogrammed end of the discharge. The remnant heat load on the targets is $7 \%$ of the $3 \mathrm{MW}$ ECR-heating power including the contributions of photons and chargeexchange neutrals. This implies that, at least $93 \%$ of the total power is removed before reaching the targets. This result is consistent with the bolometer results within error bars, presenting a good power balance. The effective ion charge in the bulk plasma $Z_{\text {eff }}$ (figure 2), as determined from bremsstrahlung radiation, is 3 before pellet injection (with an uncertainty of 1); it has a slight increment after pellet injection approaching 3.5 in the detachment phase. This variation may indicate that impurity transport is partially involved in establishing the regime, which is a topic requiring further studies.

In the transient phase from $t=1.7$ to $3.0 \mathrm{~s}$, two maxima of $P_{\text {rad }}$ (figure 2) have been obtained: one at $t=2.1 \mathrm{~s}$ corresponding to the maximum density and the other at $t=2.7 \mathrm{~s}$ (with $P_{\text {rad }}>P_{\mathrm{ECRH}}$ ) associated with the enhancement of $Z_{\text {eff }}$ and the decline of $W_{\mathrm{P}}$.

It is noteworthy that the Thomson scattering measurements (together with Gaussian process fits[33], i.e. nonparametric fits of the profiles through covariance functions carried out within the Minerva Bayesian modelling framework[30]) shown in figure 3 demonstrate an edgedensity increment at $t=3.5 \mathrm{~s}$ (in red). A rise of $n_{\mathrm{e}, \mathrm{LCFS}}$ up to $1.7 \pm 0.310^{19} \mathrm{~m}^{-3}$ with a flatter density profile in comparison to that at $t=1.7 \mathrm{~s}$ is observed. The electron temperature has a slight reduction at the LCFS, comparable, however, to the diagnostic uncertainty of about 20 $\mathrm{eV}$.

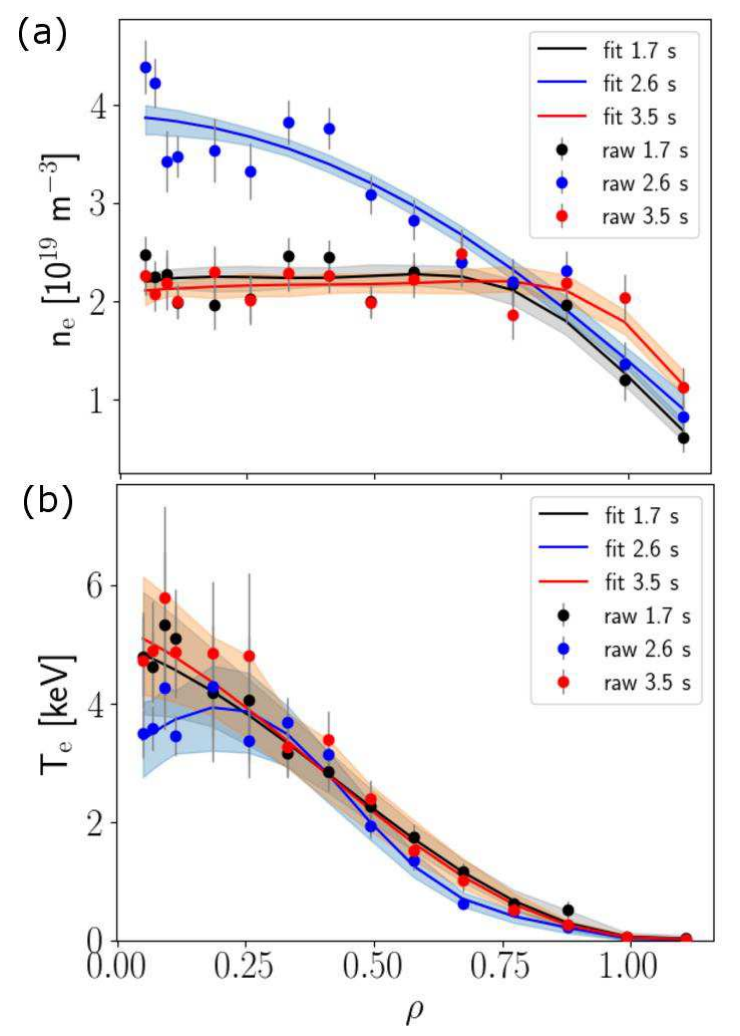

FIG. 3. (a) Electron density and (b) temperature profiles measured with Thomson scattering (solid points) at $t=1.7 \mathrm{~s}$ (before pellet injection), $2.6 \mathrm{~s}$ (just after pellet injection), and $3.5 \mathrm{~s}$ (in the regime) in the discharge \#20171109.045. The radial position $\rho$ marks the normalized effective radius of the magnetic flux surface and $\rho=1$ corresponds to the last closed flux surface. The solid curves are Gaussian process fits[33] to the measured data points and the shadowed region represents the standard deviation of the fits. It shows the pronounced central peaking of the density profile caused by pellet fueling followed by a recovery at the plasma center and, in particular, an increment of $n_{e, L C F S}$ at $t=3.5 \mathrm{~s}$; the temperature profile change is comparatively small, however.

Figure 4 (a) shows the corresponding temporal evolution of the chord brightness of the vertical bolometer camera (VBC). After pellet-injection $(t>2.1 \mathrm{~s})$, the brightness of the bolometer channels that detect the SOL region sharply drops due to cooling of the peripheral plasma, especially the inboard side. After detachment $(t>3.3 \mathrm{~s})$, the edge bolometer channels at or slightly outside the LCFS show the strongest signals, indicating that the radiation is mainly located at the plasma edge. This is confirmed by the data from the horizontal camera (HBC) and also a preliminary tomographic reconstruction of the radiation intensity distribution (not shown here) using both the HBC- and VBC-measurements with the Gaussian Process Tomography method[34]. Additionally, a poloidal asymmetry that has more intensive radiation from the plasma region below the midplane has been observed. This emissivity asymmetry is often observed 


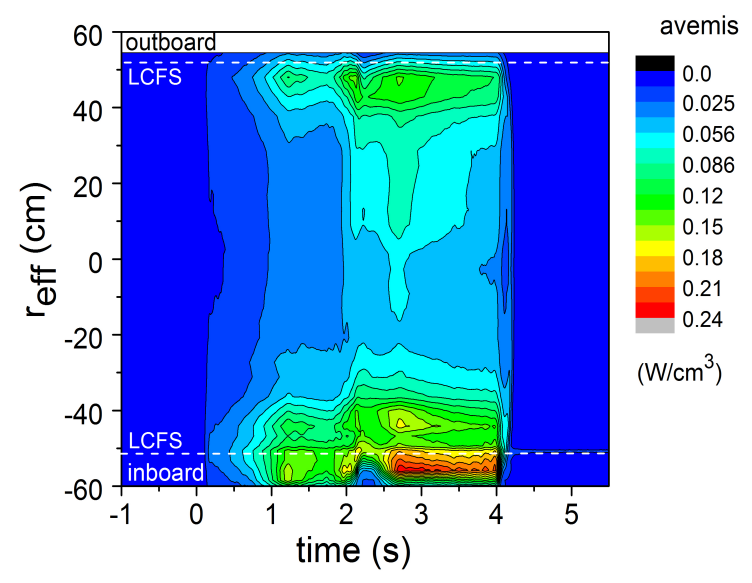

FIG. 4. Temporal evolution of the brightness of the VBC channels labeled by their tangential radii of closed flux surfaces $\left(r_{e f f}\right.$ on the vertical axis with negative numbers indicating the inboard side). They are flux surfaces in the confinement region, and linearly extended from a smooth flux surface near the separatrix into the edge islands.

for high-density, high-radiation phases. It may result from unevenly distributed impurity sources originating from plasma-surface-interaction regions. Further analysis shows that approximately $80 \%$ of the power is radiated from the near-separatrix region.

During this divertor experimental campaign carbon and oxygen have been identified as main impurity species. Their absolute concentration is however unknown. Figure 5 shows the time-traces of the line emissions from CIII $(117.5 \mathrm{~nm})$, CIV $(31.2 \mathrm{~nm})$, OV $(22.02 \mathrm{~nm})$ and OVI $(12.98 \mathrm{~nm})$. These are the most intensive radiating ion stages of carbon and oxygen and have therefore been selected as representative for the total carbon and oxygen radiation. The temporal evolution of $P_{\mathrm{rad}}$ is compared with that of the four selected impurity ion emissions.

For this purpose, each line emission curve is aligned with the normalized $P_{\text {rad }}$ at $t=1.7 \mathrm{~s}$ (just before the pellet injection). The measured line-emissions from OV and OVI follow well the time evolution of $P_{\text {rad }}$, whereas those from CIII and CIV do not. In particular, the CIII and CIV line emission intensities do not rise in the high radiation phase after the pellet injection; the CIV brightness even sharply drops. (Additional analysis treating line emissions from impurity ions with even lower ionization stages, such as CII and OIII, close to the PSI region also indicates no correlation with the $P_{\text {rad }}$ evolution). Considering that CIV is the ionization stage of carbon that radiates most effectively, the comparison suggests a dominant role of oxygen in contributing to the total radiation. This is likely due to the high radiation potential of oxygen, which is about three times larger than that of carbon[35], but

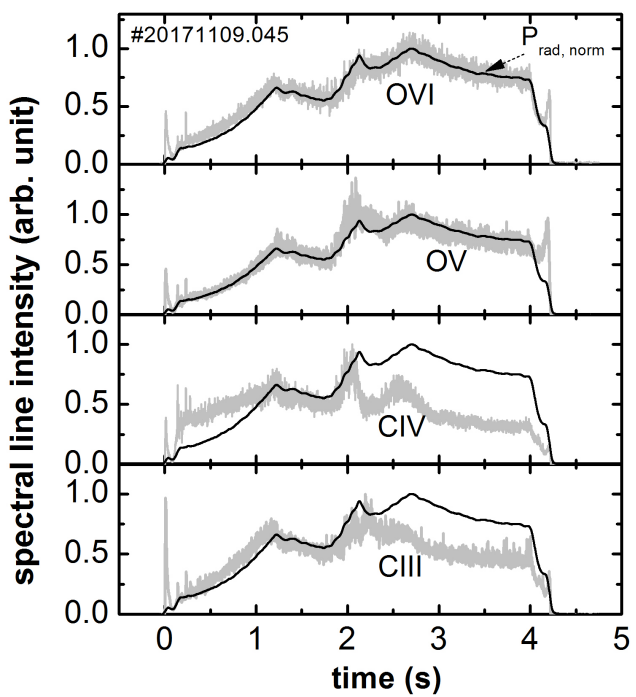

FIG. 5. Line-radiation of OVI, OV, CIV and CIII for the same discharge as shown in Figs. 2-4. For comparison $P_{\text {rad }}$ is shown as black lines.

could also be due to a higher oxygen influx. The impurity influx is not known in this discharge. However, according to the mass spectrometry data, relatively high $\mathrm{H}_{2} \mathrm{O}$ and $\mathrm{CO}$ partial pressure has been measured in this discharge. Clarification of the origins and recycling processes of oxygen impurities in W7-X is beyond the scope of this paper.

In summary, the first island divertor experiments on W7$\mathrm{X}$ have demonstrated that a stable highly-radiative divertor plasma regime can be established. Oxygen radiation, which is located at the edge plasma region, plays a key role. Strong reduction of heat flux on the divertor targets shows plasma detachment although the plasma stored energy is well-maintained. This regime was first observed in the studied discharge with pellet injection, and was later found in discharges fueled via gas-puff and even in discharges with only a prefill. A certain amount of particle fueling is needed to provide the necessary recycling and plasma conditions in the scape-off layer, while the fueling location does not seem to play a role. In this regime, two target Langmuir probe arrays, which were out of operation in the discharge mentioned above (\#20171109.045) but available for other relevant discharges, have measured significantly reduced ion saturation currents indicating strongly reduced particle flux. Nevertheless, there is no experimental evidence for noticeable volume recombination, so far. The fact that the heat flux drops uniformly over the wetted area on the targets at W7-X shows that the island divertor concept at W7-X performs as desired and providing good power dissipation, yielding a promising exhaust option for the stellarator reactor line.

This work has been carried out within the framework of the EUROfusion Consortium and has received funding from the Euratom research and training pro- 
gramme 2014-2018 and 2019-2020 under grant agreement No 633053. The views and opinions expressed herein do not necessarily reflect those of the European Commission.

[1] T. Petrie et al., Journal of Nuclear Materials 196-198, 848 (1992), plasma-Surface Interactions in Controlled Fusion Devices.

[2] K. Lackner and R. Schneider, Fusion engineering and design 22, 107 (1993).

[3] O. Gruber et al., Physical review letters 74, 4217 (1995).

[4] G. Matthews, Journal of nuclear materials 220, 104 (1995).

[5] B. Lipschultz et al., Journal of nuclear materials 220, 50 (1995).

[6] A. Kallenbach et al., Nuclear Fusion 55, 053026 (2015).

[7] Y. Feng et al., Nuclear Fusion 56, 126011 (2016).

[8] M. Bernert et al., Nuclear Materials and Energy 12, 111 (2017).

[9] F. Karger and K. Lackner, Physics Letters A 61, 385 (1977).

[10] T. Evans et al., Journal of Nuclear Materials 145-147, 812 (1987).

[11] T. Evans et al., Journal of Nuclear Materials s 162-164, 636-642 (1989).

[12] H. Renner, Plasma Phys. Controlled Fusion 31, 1579 (1989).

[13] C. Beidler et al., Fusion Technology 17, 148 (1990).

[14] H.-S. Bosch et al., Nuclear Fusion 53, 126001 (2013).

[15] P. Grigull et al., Plasma physics and controlled fusion 43, A175 (2001).

[16] K. McCormick, P. Grigull, R. Burhenn, et al., Phys. Rev. Lett. 89, 015001 (2002).

[17] L. Giannone, R. Burhenn, K. McCormick, et al., Plasma physics and controlled fusion 44, 2149 (2002).
[18] M. Hirsch et al., Plasma Physics and Controlled Fusion 50, 053001 (2008).

[19] J. Nührenberg, Plasma Physics and Controlled Fusion 52, 124003 (2010).

[20] R. C. Wolf et al., Plasma and Fusion Research 5, S1011 (2010).

[21] T. Klinger et al., Plasma Physics and Controlled Fusion 59, 014018 (2016).

[22] T. Sunn Pedersen et al., Physics of plasmas 24, 055503 (2017).

[23] A. Dinklage et al., Nature Physics 14, 855 (2018).

[24] Y. Feng, F. Sardei, P. Grigull, K. McCormick, J. Kisslinger, and D. Reiter, Nuclear fusion 46, 807 (2006).

[25] D. Zhang, R. Burhenn, R. König, L. Giannone, P. Grodzki, et al., Review of Scientific Instruments 81, 10E134 (2010).

[26] Y. Feng, F. Sardei, J. Kisslinger, P. Grigull, K. McCormick, and D. Reiter, Contributions to Plasma Physics 44, 57 (2004).

[27] M. Krychowiak et al., Review of Scientific Instruments 87, 11D304 (2016).

[28] K. Rahbarnia, H. Thomsen, U. Neuner, et al., Nuclear Fusion 58, 096010 (2018).

[29] M. Jakubowski et al., Review of Scientific Instruments 89, 10E116 (2018).

[30] J. Svensson and A. Werner, in 2007 IEEE International Symposium on Intelligent Signal Processing (IEEE, 2007) pp. 1-6.

[31] N. Ohyabu et al., Physical review letters 97, 055002 (2006).

[32] T. Morisaki et al., Physics of plasmas 14, 056113 (2007).

[33] C. E. Rasmussen and C. K. I. Williams, Gaussian Processes for Machine Learning (The MIT Press, 2006).

[34] J. Svensson, JET Internal report, Tech. Rep. (EFDAJET-PR (11) 24, 2011).

[35] U. Samm et al., Journal of Nuclear Materials 176, 273 (1990). 